\title{
III-Nitride-Based Planar Lightwave Circuits for Long Wavelength Optical Communications
}

\author{
Rongqing Hui, Senior Member, IEEE, Yueting Wan, Jing Li, Sixuan Jin, Jingyu Lin, and Hongxing Jiang
}

\begin{abstract}
Planar lightwave circuits based on III-nitride widebandgap semiconductors are proposed and the feasibility of developing III-nitride-based novel photonic integrated circuits for applications in fiber-optical communications is discussed. III-nitrides have low attenuation in the near-infrared wavelength region because of their wide bandgaps, while as semiconductors their refractive indexes can be modulated by carrier injection. III-nitrides are also well known for their ability to operate at high temperatures, high power levels and in harsh environments. These characteristics make III-nitrides ideal candidates for tunable optical phased-array (PHASAR) devices for optical communications. We have characterized the optical properties of $\mathrm{Al}_{x} \mathrm{Ga}_{1-x} \mathrm{~N}$ epilayers in the $1550-\mathrm{nm}$ wavelength region, including the refractive indexes and the impact of Al concentrations. Single-mode ridged optical waveguide devices using GaN-AIGaN heterostructures have been designed, fabricated and characterized for operation in the $1550-\mathrm{nm}$ wavelength window. The birefringence of wurtzite GaN grown on sapphire substrate has been observed. Refractive indexes were found to be different for signal optical field perpendicular and parallel to the crystal $c$ axis $\left(n_{\perp} \neq n_{/ /}\right)$. More importantly, we found an approximately $10 \%$ change in the index difference $\Delta n=n_{/ /}-$ $n_{\perp}$ with varying the waveguide orientation within the $c$ plane, and a $60^{\circ}$ periodicity was clearly observed. This is attributed to the hexagonal structure of the nitride materials. Various functional waveguide devices have been realized, including $2 \times 2$ directional couplers and eight-wavelength array-waveguide gratings. Theoretical predictions of temperature sensitivity and the efficiency of carrier-induced refractive change are provided.
\end{abstract}

Index Terms-Integrated optics, optical communication, planar waveguides, semiconductor devices, semiconductor materials, semiconductor waveguides, wavelength division multiplexing.

\section{INTRODUCTION}

$\mathbf{I}$ $\mathrm{N}$ ORDER to make full use of the wide bandwidth provided by optical fibers, more and more wavelength channels have been used in wavelength division multiplexed (WDM) optical networks. Among others, WDM optical demultiplexers, optical switches and wavelength routers are fundamental devices in multiwavelength, dynamic optical networks. With the rapid advancement of photonic lightwave circuits (PLC), silicabased array waveguide grating (AWG) has become a popular

Manuscript received June 17, 2004; revised September 21, 2004. This work was supported in part by the National Science Foundation through "Ultra-HighCapacity Optical Communications and Networks" under Grants ECS-0 123 450, DMR-0203373, and ARO (DAAD19-03-1-0337).

R. Hui and Y. Wan are with the Department of Electrical Engineering and Computer Science, University of Kansas, Lawrence, KS 66044 USA (e-mail: rhui@ku.edu; ywan1@ittc.ku.edu).

J. Li, S. Jin, J. Lin, and H. Jiang are with the Department of Physics, Kansas State University, Manhattan, KS 66506-2601 USA (e-mail: jingli@phys.ksu.edu; sixjin@phys.ksu.edu; jylin@phys.ksu.edu; Jiang@ phys.ksu.edu).

Digital Object Identifier 10.1109/JQE.2004.838169 approach for WDM multiplexing and demultiplexing [1]. Since silica is not a semiconductor material and hence purely passive, the transfer functions of silica-based AWGs are usually not tunable, or can only be slowly tuned by thermal effect [2], [3]. InP has been another popular material to make PLCs. As a semiconductor material, InP-based PLCs can potentially be made fast tunable with carrier injection [4]; however, due to its high refractive index, high temperature sensitivity and high insertion loss [5], InP-based AWG devices have, so far, not become commercially competitive.

III-nitride wide bandgap semiconductor materials have attracted considerable interest in recent years [6], [7]. In addition to emitting light in ultraviolet (UV)/blue wavelength region, III-nitride optoelectronic devices are able to operate at high temperatures and high power levels due to their mechanical hardness and large band offsets. So far, research in III-nitrides has been focused on applications in the blue/UV wavelength regions. Their optical characteristics in near infrared wavelengths remain largely unknown and their potential application in fiber-optical communications has not been fully investigated. In this paper, we propose to make functional optical waveguide devices using $\mathrm{GaN}-\mathrm{AlGaN}$ semiconductor materials and explore their potential applications in infrared wavelength regions for fiber-optic communications. We found that the refractive index of GaN decreases with wavelength and reaches to approximately 2.31 in the $1550-\mathrm{nm}$ wavelength window [8]. This provides a much better refractive index match with optical fibers than InP does. Furthermore, the refractive index of $\mathrm{Al}_{x} \mathrm{Ga}_{1-x} \mathrm{~N}$ was found to decrease monotonically with the increase of $\mathrm{Al}$ concentration, which permits a precise index adjustment that is critical in integrated optical circuit design. More importantly, since III-nitrides are semiconductor materials, in addition to making passive devices such as fixed-wavelength filters, splitters and AWGs, they may also enable active functionalities such as tunable filtering and photonic switches. Dynamic switch and tuning can be accomplished by carrier-induced refractive index change. Due to their mechanical hardness and large band gaps, III-nitride based devices may operate at much higher optical power levels for any dimensional configuration. Previous research indicated that III-nitrides have very low temperature sensitivity, especially in infrared wavelength regions [9]. This is another crucial advantage III-nitrides have for applications in optical communications.

\section{MEASUREMENT OF REFRACTIVE INDICES IN INFRARED}

In order to design guided-wave optical devices, the knowledge of material refractive indexes in the operating wavelength 


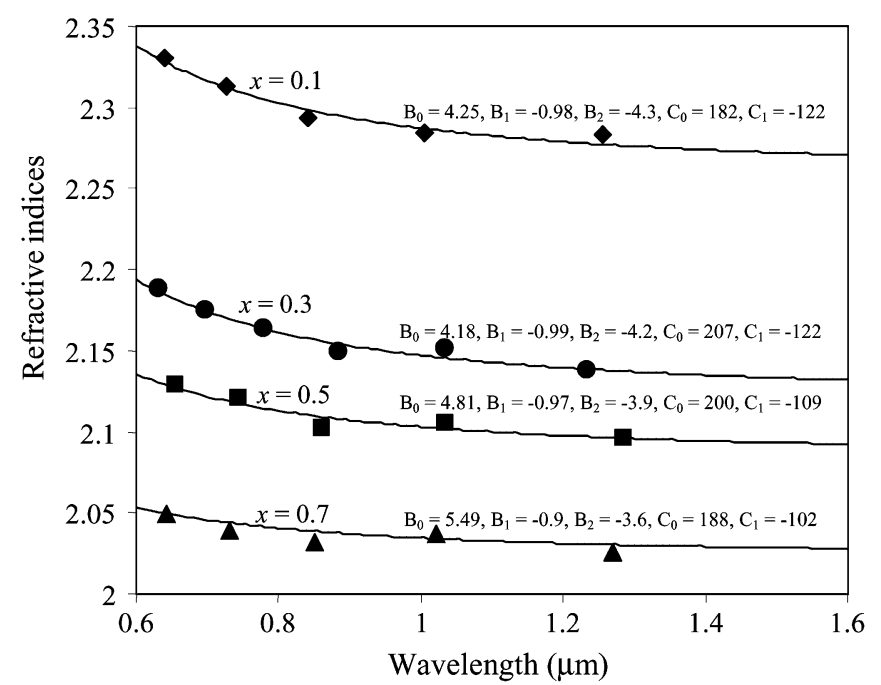

(a)

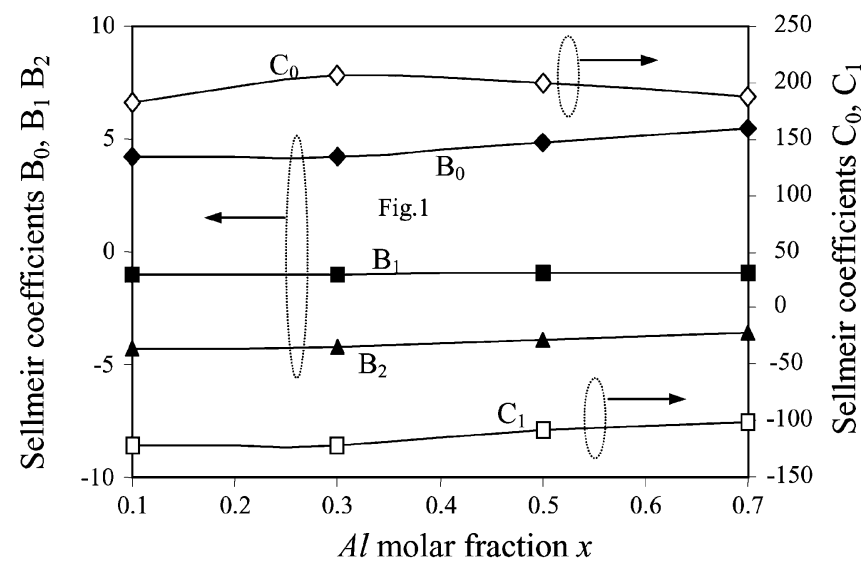

(b)

Fig. 1. Refractive indexes of $\mathrm{Al}_{x} \mathrm{Ga}_{1-x} \mathrm{~N}$ versus wavelength for several different Al molar fractions. Solid points: measured. Continuous curves: numerical fittings using (1). Fitting parameters are listed beside each curve.

region is essential. We have conducted the refractive index measurements for $\mathrm{Al}_{x} \mathrm{Ga}_{1-x} \mathrm{~N}$ with different $\mathrm{Al}$ molar fractions. In order to perform this measurement, a number of sample $\mathrm{Al}_{x} \mathrm{Ga}_{1-x} \mathrm{~N}$ films were grown by metal-organic chemical vapor deposition (MOCVD) on sapphire substrates. The films' thickness range from 1.1 to $1.5 \mu \mathrm{m}$ and $\mathrm{Al}$ molar fractions range from $x=0.1$ to $x=0.7$ [10]. To evaluate the refractive index of each film, optical transmission spectra were measured. Due to the Fabry-Perot (FP) interference caused by the two facets of the film (one facet is between $\mathrm{Al}_{x} \mathrm{Ga}_{1-x} \mathrm{~N}$ and the air and the other facet is formed between $\mathrm{Al}_{x} \mathrm{Ga}_{1-x} \mathrm{~N}$ and sapphire), optical transmission efficiency is wavelength-dependent. With the knowledge of the film thickness, the film refractive index can be obtained by best fitting the measured optical transmission spectrum to a well-known FP transmission equation.

Fig. 1(a) shows the measured refractive indexes of $\mathrm{Al}_{x} \mathrm{Ga}_{1-x} \mathrm{~N}$ versus wavelength for several different $\mathrm{Al}$ molar fractions. The continuous curves in the same figure were numerical fittings obtained by using the first-order Sellmeir dispersion formula

$$
n(\lambda)=\sqrt{1+\frac{\left(B_{0}+B_{1} x+B_{2} x^{2}\right) \lambda^{2}}{\lambda^{2}-\left(C_{0}+C_{1} x\right)^{2}}} .
$$

The coefficients for best fit are displayed in Fig. 1(a) and their variations versus Al molar fraction $x$ are shown in Fig. 1(b). The measured refractive indexes versus wavelength are in good agreement with the previously reported values [11]. Since we are mostly interested in the refractive indexes in 1550-nm wavelength window, this information can be collected from Fig. 1 and the following polynomial expression is obtained for the Al molar fraction $(x)$ dependence of the refractive index at 1550-nm wavelength:

$$
n(1550 \mathrm{~nm})=0.431 x^{2}-0.735 x+2.335 .
$$

The monotonic decrease of $\mathrm{Al}_{x} \mathrm{Ga}_{1-x} \mathrm{~N}$ refractive index with the increase of Al molar fraction $x$ makes the design of singlemode optical waveguide devices straightforward.

\section{Optical WaVEGuide PREPARATION AND CHARACTERIZATION}

We have used beam propagation method (BPM) simulation tools to design single-mode optical waveguide devices. Fig. 2 schematically shows the cross section of the designed single-mode waveguide based on $\mathrm{GaN}$ core and $\mathrm{Al}_{x} \mathrm{Ga}_{1-x} \mathrm{~N}$ cladding. Several different waveguide configurations have been designed, including straight waveguides and $2 \times 2$ waveguide couplers. To verify the design, a number of waveguide samples were prepared. In the fabrication process, a 4 - $\mu \mathrm{m}$-thick epitaxial film of $\mathrm{Al}_{x} \mathrm{Ga}_{1-x} \mathrm{~N}$ was grown on a sapphire substrate and a 3- $\mu$ m-thick GaN film was deposited on top of the $\mathrm{Al}_{x} \mathrm{Ga}_{1-x} \mathrm{~N}$ layer. Then, the optical waveguide structures were formed by photolithographic patterning and inductively coupled plasma (ICP) dry etching [7]. According to the design, the etching depth is controlled at approximately $2.8 \mu \mathrm{m}$ and the Al molar fraction $x$ is about $3 \%$. As an example, Fig. 3 shows a typical $2 \times 2$ waveguide coupler fabricated with this process. The power splitting ratio of this particular coupler was designed to be $3 \mathrm{~dB}$.

To characterize the waveguide samples, a fiber-optic setup operating in the $1550-\mathrm{nm}$ wavelength region was used. Optical coupling at the input and the output of the waveguide was accomplished by using tapered single-mode fibers with $6-\mu \mathrm{m}$ working distance and $2.5-\mu \mathrm{m}$ spot size of the focus. Each tapered fiber end was mounted on a five-dimensional precision positioning-stage to optimize the optical coupling efficiency. A tunable laser diode was used as a light source and an optical power meter was used to measure the optical power that passes through the waveguide. Fig. 4 shows the measured optical power versus the horizontal position of the tapered output fiber probe. In this measurement, the input fiber probe was aligned with one of the two input ports of the waveguide coupler. This figure clearly shows an approximately $50 \%$ power splitting realized by this $\mathrm{GaN}-\mathrm{AlGaN}$ waveguide coupler, thus validating our original design. In order to evaluate the loss of the waveguide, we have measured a 1.395-mm-long straight waveguide. The experiment was based on the measurement of FP interference caused by the Fresnel reflections of the two end facets of the waveguide. This excluded the uncertainties due to optical coupling efficiencies at the input and output of the waveguide. An erbium-doped fiber amplifier (EDFA) without 


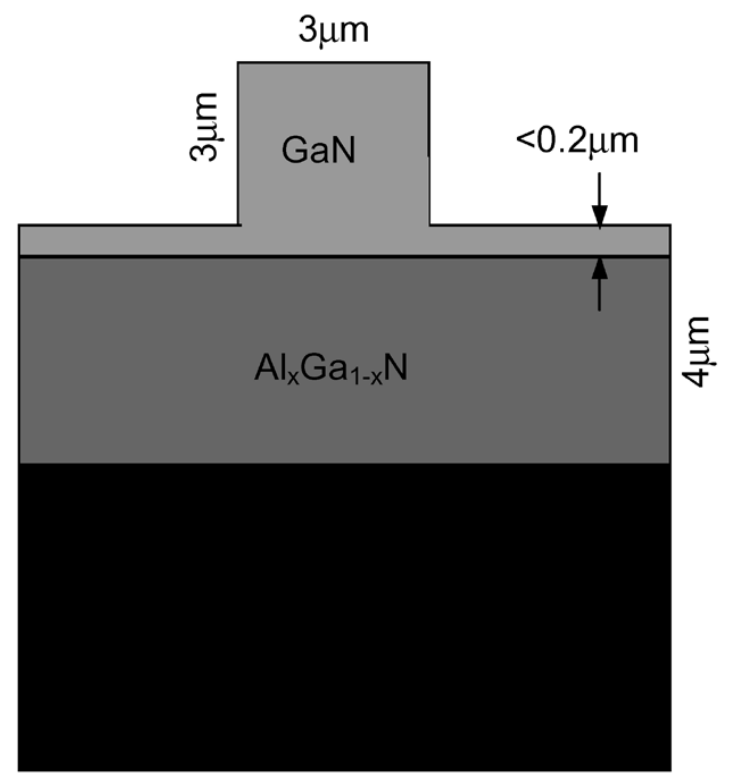

(a)

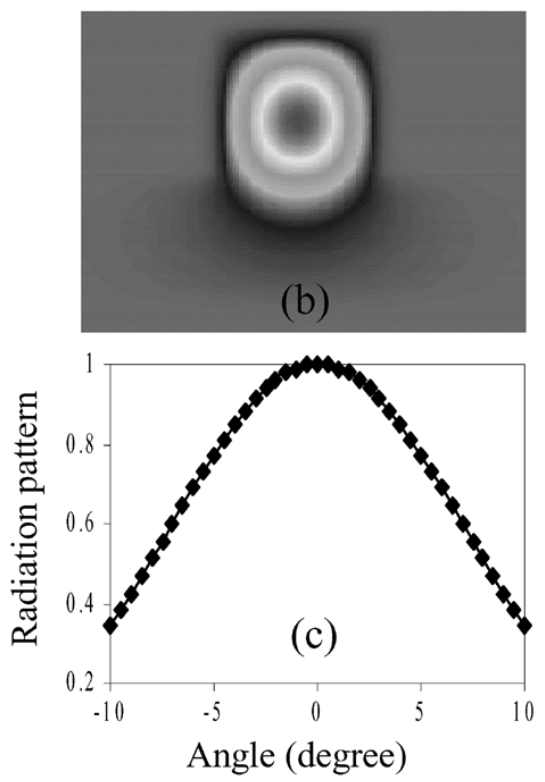

Angle (degree)

Fig. 2. Optical waveguide design using $\mathrm{GaN}-\mathrm{Al}_{x} \mathrm{Ga}_{1-x} \mathrm{~N}$ on sapphire. (a) Waveguide cross section. (b) Simulated single-mode structure. (c) Radiation pattern versus exit angle.

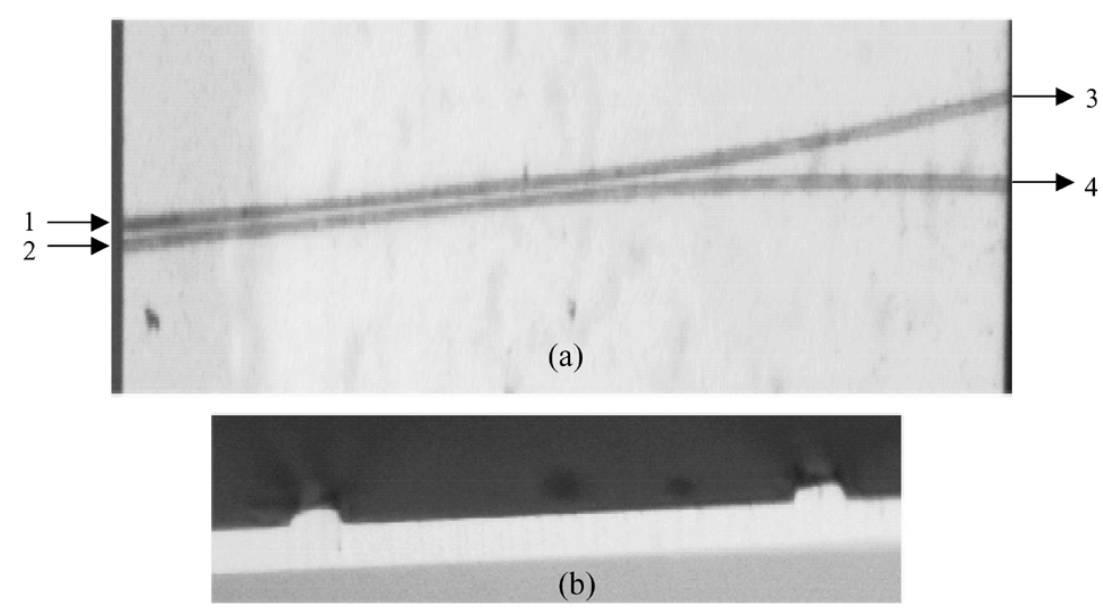

Fig. 3. Photograph of a $2 \times 2 \mathrm{GaN}-\mathrm{AlGaN}$ heterostructure optical waveguide coupler. (a) Top view. (b) Cross-section view at the output.

optical signal input was used in this measurement, which provides a wideband amplified spontaneous emission (ASE). An optical spectrum analyzer was used to measure the ASE optical spectrum after it passed through the optical waveguide sample. The solid points in Fig. 5 show the measured optical spectrum which was normalized such that the average power spectral density is at $0-\mathrm{dB}$. The continuous line in the same figure was calculated by using the normalized FP transmission equation

$$
T(\lambda)=\left\{1+R^{2} A^{2}-2 R A \cos \left(4 \pi L n_{\mathrm{eff}} / \lambda+\varphi_{0}\right)\right\}^{-1}
$$

where $L=1.395 \mathrm{~mm}$ is the waveguide length, $R=$ $\left[\left(n_{\text {core }}-1\right) /\left(n_{\text {core }}+1\right)\right]^{2}=16.02 \%$ is the power reflectivity of the waveguide end surface, $\varphi_{0}$ is an initial phase which can be determined by the best fitting between the measured and the calculated spectrum, and $A$ is the single-pass power attenuation through the waveguide. $n_{\text {eff }}$ is the effective index of the waveguide. Since the refractive indexes for the core and the cladding are $n_{\text {core }}=2.335$ and $n_{\text {clading }}=2.275$, respectively, with the waveguide geometry shown in Fig. 2, the effective index can be determined as $n_{\text {eff }}=2.315$ through a BPM simulation. To obtain the best fitting to the measured spectrum as shown in Fig. 5, $A=4.8 \mathrm{~dB}$ was used. It is worth mentioning that in sample preparation, the cleaved waveguide end facets are not ideally flat and they are not exactly perpendicular to the waveguide axis either; therefore, the effective facet reflectivity is less than $16.02 \%$. For this reason, we expect that the actual attenuation of the waveguide should be significantly less than $4.8 \mathrm{~dB}$. Other optical loss mechanisms such as the scattering at the waveguide edge can be further reduced by improving the etching process.

\section{BIREFRINGENCE OF GaN-AlGaN OptiCAL WAVEGUIDES}

In planar lightwave circuit applications, the refractive index of the material is an important parameter as described in the pre- 


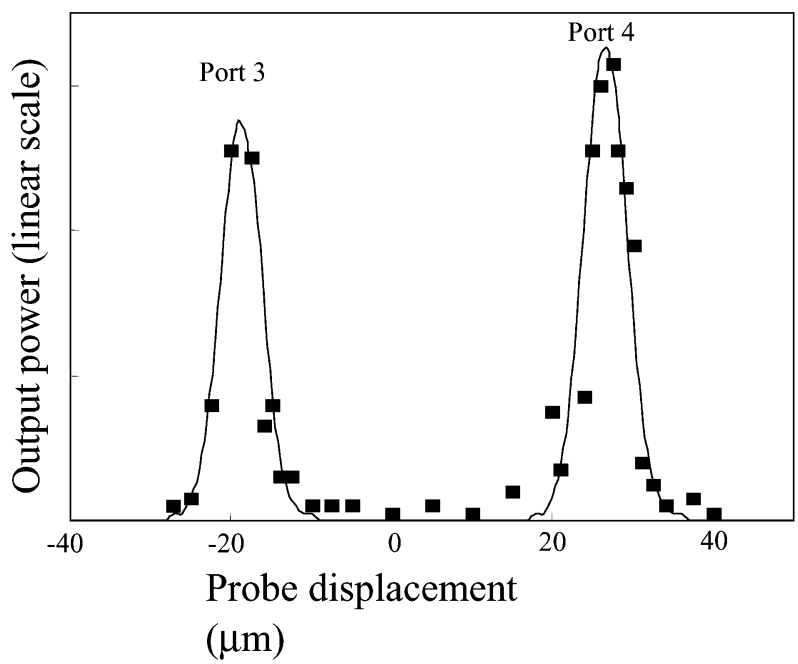

Fig. 4. Measured optical power versus output fiber probe displacement in the horizontal direction. The input optical signal is launched at port 1 (as illustrated in Fig. 3),

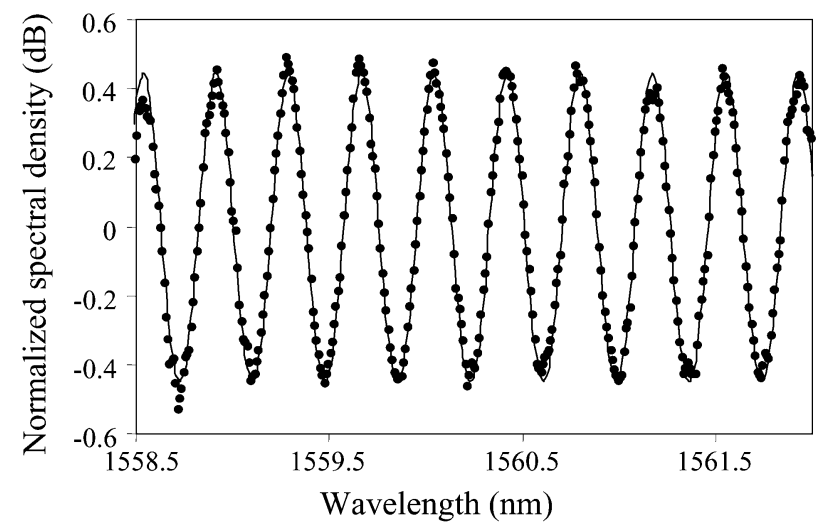

Fig. 5. Measured optical transmission spectrum (solid points) and numerical fitting using (3) (continuous line). Fitting parameters used: $n_{\text {eff }}=2.315$ and $A=4.8 \mathrm{~dB}$.

vious section. In addition, the knowledge of the birefringence of the material is also critical in waveguide design and device performance. The birefringence of GaN films grown on sapphire substrates has been previously reported [12], where a refractive index change of approximately $4 \%$ was observed in the long wavelength region (around $800 \mathrm{~nm}$ ) when the signal polarization was switched from perpendicular to the crystal $c$ axis to parallel [13]. On the other hand, due to the wurtzite structure, the crystal lattice of $\mathrm{GaN}$ has a hexagonal configuration on the $c$ plane. It has been shown that the efficiency of optical emission changed periodically when the optical propagation direction was varied on the $c$ plane [14]. We have investigated the birefringence of single-mode $\mathrm{GaN}-\mathrm{AlGaN}$ optical waveguides operating in 1550-nm optical communication wavelength region. We found that the refractive index not only depends on the signal polarization states (parallel or perpendicular to the $c$ axis), it also depends on the direction of optical propagation within the $c$ plane.

Fig. 6 schematically shows the cross section and the crystal $(a, b, c)$ coordinate of the designed optical waveguide based on $\mathrm{GaN}$ core, $\mathrm{Al}_{x} \mathrm{Ga}_{1-x} \mathrm{~N}$ cladding and sapphire substrate. In order to investigate the birefringence effect as a function of wave propagation direction, a number of waveguide samples were pre-

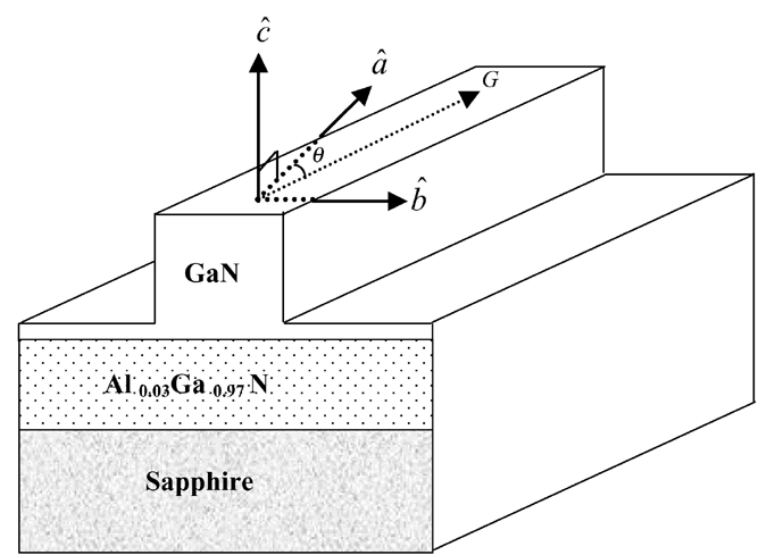

Fig. 6. Schematic illustration of waveguide cross section and wave propagation direction $G$ with respective to $\mathrm{GaN}$ crystal structure in the Cartesian-coordinates $(c \perp a \perp b)$. The waveguide orientation angle $\theta$ is defined as the angle between $G$ and $a$ within the $a-b$ plane.

pared each having a different orientation angle $\theta$ with respect to the $a$ axis within the $c$ plane. The lengths of the waveguide samples ranged from 1.5 to $3 \mathrm{~mm}$.

Similar to the previous section, this experiment was based on the measurement of FP interference caused by the Fresnel reflections from the two end facets of the waveguide. However, to evaluate the polarization effect, a fiber-optic polarizer was added immediately after the wideband ASE source and a polarization controller was inserted after the polarizer to adjust the state of signal polarization before it is injected into the waveguide sample. Fig. 7 shows an example of the measured optical transfer functions on a waveguide sample. This particular waveguide was fabricated at an azimuthal angle $\theta=15^{\circ}$ with respect to the $a$ axis of the crystal. The three traces in this figure represent the transfer functions measured with three different optical signal polarization states. Trace 1 has the input optical signal polarized perpendicular to the $c$ axis while trace 3 has the signal parallel to the $c$ axis. The average values of the transfer functions shown in the figure were intentionally shifted by $\pm 0.8 \mathrm{~dB}$ for the purpose of better display in the figure. Regular FP-like transfer functions are clearly shown by both of these two traces except for a slight difference in their period. These two traces can be numerically fit by a normalized FP transfer function

$$
T(\lambda)=\left\{1+R^{2}-2 R \cos \left[\frac{4 \pi L\left(n_{\mathrm{eff}} \pm \Delta n / 2\right)}{\lambda}+\varphi_{0}\right]\right\}^{-1}
$$

where $L=2.31 \mathrm{~mm}$ is the length of the waveguide and $R=$ $3.75 \%$ is the effective round-trip power loss of the waveguide including the mirror loss at the waveguide end surface due to Fresnel reflection. $\varphi_{0}$ is an initial phase, which can be determined by the best fitting between the measured and the calculated transfer functions. Consider the effect of birefringence, the effective refractive index is $n_{\text {eff }}=n_{\perp}$ if the optical field is perpendicular to the material $c$ axis and $n_{\text {eff }}=n_{/ /}$when the field is parallel to the $c$ axis, where generally $n_{\perp} \neq n_{/ /}$. The solid lines in Fig. 7 are numerical fittings using (4). To obtain the best fitting to the measured transfer functions (solid dots) of traces 1 and 3 , refractive indexes of $n_{/ /}=2.357$ and $n_{\perp}=2.315$ were used 


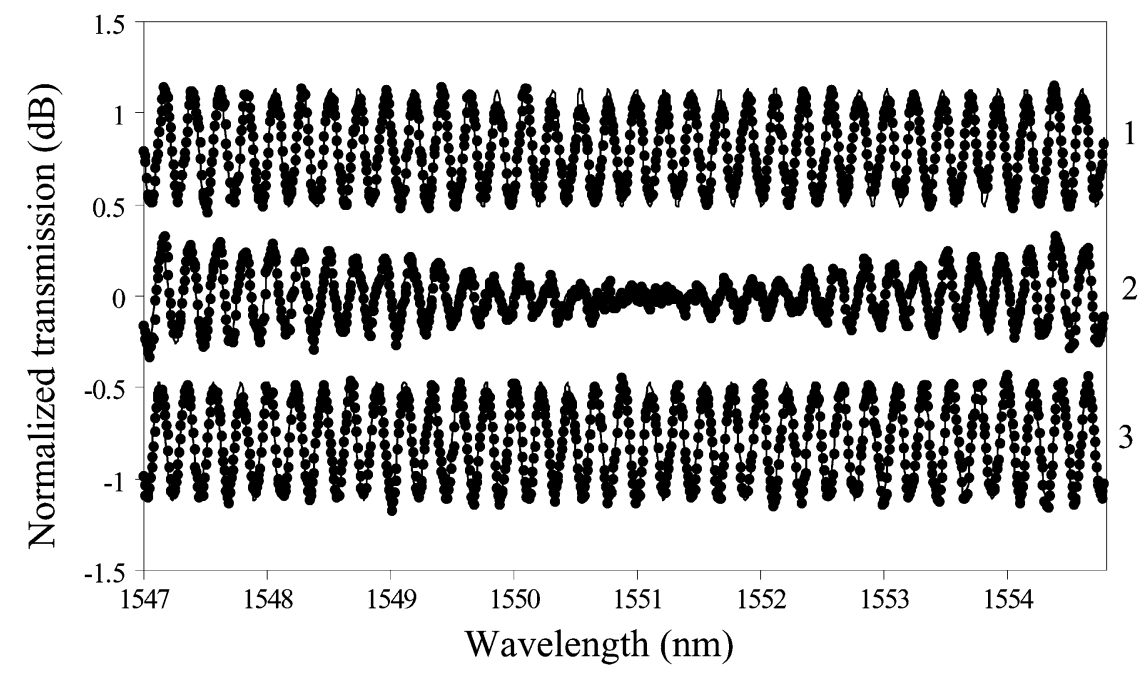

Fig. 7. Measured (solid dots) and calculated (continuous lines) optical transfer function with the input optical field perpendicular (trace 1), parallel (trace 3) and $45^{\circ}$ from (trace 2 ) the crystal $c$ axis.

and thus the value of birefringence is $\Delta n=\left(n_{/ /}-n_{\perp}\right)=0.042$ in this particular case. A separate BPM simulation indicated that with the waveguide design as shown in Fig. 2, the index difference between $\perp$ and // polarization components induced by waveguide structure is less than $10^{-5}$, which is three orders of magnitude lower than the measured birefringence. Therefore, this $\Delta n$ value of 0.042 obtained in the measurement is predominantly caused by the material birefringence. Trace 2 in Fig. 4 was obtained by splitting the input optical signal equally into $\perp$ and // polarization modes. In this case, the ripple amplitude of the FP transfer function has a null at $1551.2 \mathrm{~nm}$. This was because of a $\pi$ phase walk-off between the TE and the TM polarization components happened at that wavelength, which can be verified by a careful comparison between trace 1 and trace 3 in the same figure. We were also able to find multiple nulls in each FP transfer function by enlarging the measurement wavelength coverage. The birefringence can then be easily determined by

$$
\Delta n=\lambda_{0}^{2} /(2 L \Delta \lambda)
$$

where $\lambda_{0}$ is the average wavelength used in the measurement and $\Delta \lambda$ is the wavelength difference between the adjacent nulls in the transfer function.

A more systematic measurement was then performed by measuring a number of waveguide samples which are oriented at the angles of $\theta=0^{\circ}, 15^{\circ}, 30^{\circ}, 60^{\circ}$, and $90^{\circ}$, with respective to the $a$ axis. Fig. 8 shows the measured birefringence versus the waveguide orientation angle $\theta$. The birefringence value $\Delta n$ changed for more than $10 \%$ with varying waveguide orientation angle and, in addition, a $60^{\circ}$ periodicity was clearly observed. The continuous curve in Fig. 8 is a sinusoid fitting using $\Delta n=0.04275-0.00545 \cos (6 \theta)$. This anisotropy is most likely caused by the hexagonal crystal structure of GaN. Although the periodical variation of $\Delta n$ within $c$ plane is measured in infrared wavelengths, we believe this $60^{\circ}$ periodicity in $\Delta n$ variation also exists in wavelength regions near band edge, where the periodical emission-efficiency variation versus angle $\theta$ in $c$ plane has already been reported in [14].

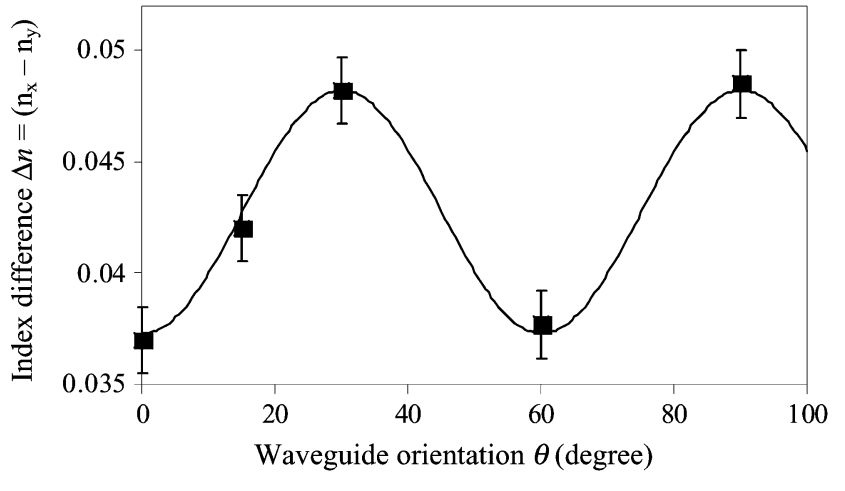

Fig. 8. Measured birefringence $\Delta n=\left(n_{/ /}-n_{\perp}\right)$ versus waveguide orientation angle $\theta$ within $a-b$ plane (solid squares). The continuous line is a sinusoid fitting using $\Delta n=0.04275-0.00545 \cos (6 \theta)$ where $\theta$ is in degree.

For most applications of planar lightwave circuits, birefringence of the waveguides has to be minimized to avoid the requirement of polarization controllers. There are a number of ways that may be used to eliminate the effect of birefringence, such as polarization compensation [15], special waveguide cross-section design [16], [17] and the introduction of built-in compressive or tensile stresses during the crystal growth [18], [19]. Among these, strain engineering has been the most popular method because it is insensitive to device configurations and functionalities. The built-in strain in the film may be caused by crystal lattice mismatch, impurity doping and dislocation. Our recent studies have indicated that the light polarization in $\mathrm{AlGaN}$ films varies with the $\mathrm{Al}$ content and that the polarization property of AlN is distinctly different from that of GaN [20]. This opens up another possibility for polarization control through bandgap engineering. Although using strain control to reduce birefringence has been reported for InGaAs-based photonic devices [18], [19], it has not been investigated in III-nitride semiconductors. There is certainly much work to be done before the creation of polarization-insensitive optical waveguide devices. 


\section{AWG BASED ON GaN-AlGaN HETEROSTRUCTURES}

AWG is one of the key optical devices in fiber-optic communications, which can be used for wavelength multiplexing, demultiplexing, and wavelength add/drop. Silica PLC is the most popular approach for AWGs and they are commercially available now. However, due to the passive nature of silica, AWG devices are usually not tunable. Thermal tuning has been used to make wavelength switches [2], [3]. In this approach, a small resistor is deposited beside each waveguide in the array so that each waveguide branch may be individually heated to introduce a relative optical delay through the thermal-induced change of the refractive index. A major limitation of this approach is the slow speed of thermal tuning in the millisecond level, which is not fast enough for packet switch-based optical networks. InP has been another popular material to make planar waveguide PLCs. As a semiconductor material, InP-based PLC can potentially be made fast tunable with carrier injection [4], [21]. However, there are several disadvantages of InP-based devices: first, its refractive index $(n \approx 3.5)$ is much higher than silica and the waveguide cross section has to be very small. The interface loss between a single-mode InP waveguide and an optical fiber is usually high because of the refractive index mismatch and the mode spot size mismatch. Secondly, the temperature sensitivity of the refractive index of InP material is in the order of $d n / d T \approx 10^{-4} \mathrm{~K}^{-1}$, which is approximately ten times higher than that of silica. The performance of InP-based AWG devices is very sensitive to the temperature change and stringent temperature control is required for this type of devices. InP-based AWG devices have so far, not become commercially competitive partly because of these reasons.

III-nitrides have distinct advantages over both silica and InP for the application of tunable AWG optical devices. On one hand, the refractive indexes of III-nitrides are less than 2.3, which is closer to that of silica and their intrinsic loss is small because their wide bandgap relative to the application wavelength of $1550 \mathrm{~nm}$. On the other hand, III-nitrides are semiconductors and PIN hererojunctions can be made, which allows carrier injection. Since the application wavelength of $1550 \mathrm{~nm}$ is far away from the material bandgap, optical gain or loss at this wavelength will not be affected significantly by the carrier density change, however, the effective refractive index will be a function of carrier injection primarily due to free carrier absorption. This allows the creation of fast tunable AWG devices. To verify the feasibility, we have designed and fabricated $\mathrm{GaN}-\mathrm{AlGaN}$-based AWG for the first time. R-Soft BPM simulation package [22] was used in the waveguide structural design and the fabrication procedure is identical to that described in Section III, that involved photolithographic patterning and ICP dry etching [7]. Fig. 9 shows an example of an AWG-based $1 \times 8$ WDM demultiplexer. Fig. 9(a) is a microscope picture of the device configuration, which consists of 1 input waveguide, 8 output waveguides, 2 star couplers, and 40 arrayed waveguides between the 2 star couplers. By design, the channel spacing of the WDM outputs is $2 \mathrm{~nm}$. Fig. 9(b) shows the measured optical transfer function versus the signal wavelength for all the 8 output ports. The results clearly demonstrated an approximately 2-nm wavelength spacing between transmission peaks of adjacent output

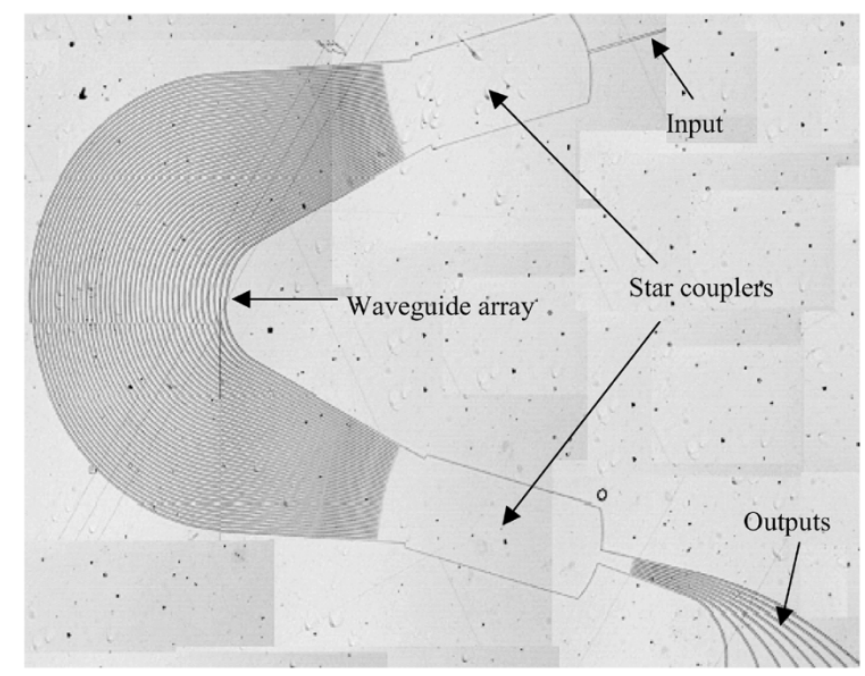

(a)

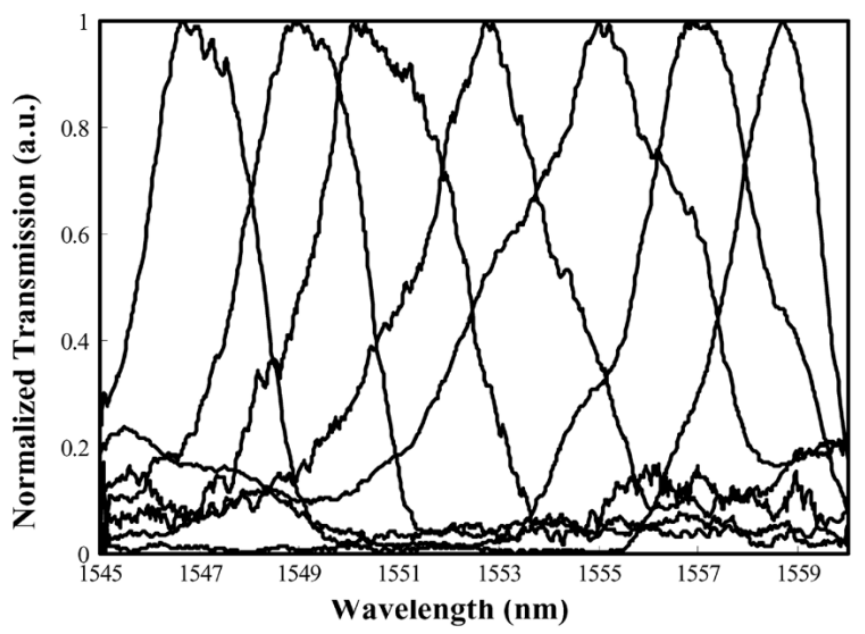

(b)

Fig. 9. (a) Microscope picture and (b) measured optical transfer function of an AWG-based WDM coupler with 2-nm channel separation.

waveguides. This indeed verifies the accuracy of the design and the single-mode operation of the waveguides. It is noticed that the out-of-band rejection ratio of the measured transfer function is only approximately ten at most of the output ports. This is mainly due to the nonuniformity of the waveguide array caused by the imperfections in the fabrication process. In the future, we will employ patterned sapphire substrates to provide suspended lateral growth to reduce dislocation density [23]-[25]. A high quality AlN epilayer can be added between the sapphire substrate and subsequent device layers to reduce the lattice mismatch, to provide an atomic flat template, and to act as dislocation filter [26], [27]. To reduce the effect of optical scattering due to imperfections of waveguide edges, a low index dielectric layer of $\mathrm{SiO}_{2}$ can be deposited by e-beam evaporation to cover the ridge waveguide structure and smooth out the waveguide edges. More importantly, the photolithographic patterning and etching processes will have to be further optimized, including optimizing the plasma etching rate and the use of an optimized resist (or a lift-off) material and process that will withstand the plasma etching as well as maintain the smooth feature of the waveguide structure. 


\section{THERMAL SENSITIVITY AND EFFICIENCY OF CARRIER-INDUCED INDEX CHANGE}

For optical PHASAR applications, relative optical phase delay between waveguide array elements is the key parameter which determines the optical transfer function. To make high-speed optical switches, the injection-current tunable nature of refractive index in $\mathrm{GaN}-\mathrm{AlGaN}$ heterostructure is a desired property and the tuning efficiency is a primary concern. On the other hand, the temperature sensitivity of the material refractive index should be small enough to ensure a reliable operation in practical environment.

\section{A. Thermal Stability of Refractive Index}

The dependence of the refractive index of $\mathrm{Al}_{x} \mathrm{Ga}_{1-x} \mathrm{~N}$ on temperature has been investigated extensively [9]. Although previous research has been focused on the characteristics of $\mathrm{Al}_{x} \mathrm{Ga}_{1-x} \mathrm{~N}$ in $\mathrm{UV}$ and visible wavelength region, the theoretical formulation can be extended into near infrared. We adopt the expression of refractive index as the function of temperature, wavelength and $\mathrm{Al}$ concentration for $\mathrm{Al}_{x} \mathrm{Ga}_{1-x} \mathrm{~N}$ in [9]

$$
n(\lambda, x, T)=\frac{1}{\sqrt{2}}\left\{\varepsilon_{r}+\sqrt{\varepsilon_{r}^{2}+\varepsilon_{i}^{2}}\right\}^{1 / 2}
$$

where $\varepsilon_{r}$ and $\varepsilon_{i}$ are the real and the imaginary parts of the dielectric constant $\varepsilon$, respectively, with

$$
\begin{aligned}
\varepsilon(\lambda, x, T) & =C(x, T)+\frac{A(x, T)}{E_{g}^{1.5}(x, T)} \cdot \frac{2-\sqrt{1+y}-\sqrt{1-y}}{y^{2}} \\
y & =\frac{h c / \lambda+i \Gamma(x, T)}{E_{g}(x, T)} \\
E_{g}(x, T) & =3.502+1.35 x+0.99 x^{2}-\frac{0.224}{\exp (0.386 / T)-1}
\end{aligned}
$$

$$
\begin{aligned}
C(x, T)= & 2.49+2.27 \times 10^{-3} T-1.8 \times 10^{-6} T^{2} \\
& -\left(0.74+4.61 \times 10^{-3} T-5.33 \times 10^{-6} T^{2}\right) x
\end{aligned}
$$$$
\begin{aligned}
A(x, T)= & \left\{79.3-8.37 \times 10^{-2} T-6.73 \times 10^{-5} T^{2}\right. \\
& \left.+\left(18.99+0.13 T-1.76 \times 10^{-4} T^{2}\right) x\right\} \quad \mathrm{eV}^{1.5}
\end{aligned}
$$$$
\Gamma(x, T)=\left\{-8.69+4.13 \times 10^{-2} T\right.
$$$$
\left.+(248.24-0.19 T) x^{2}\right\} \times 10^{-3} \mathrm{eV} \text {. }
$$

This approximation is valid for the $\mathrm{Al}$ concentration $x<0.64$ and the accuracy was tested against measured data obtained with a spectroscopic ellipsometor between $3.5 \mathrm{eV}$ and $1.5 \mathrm{eV}$ (850-nm wavelength). Although the accuracy of this equation in 1550-nm wavelength region has not been fully verified, at this wavelength far away from the material bandgap, no abrupt variation is expected for the material characteristics and (6)-(12) will provide a reasonably accurate estimation for temperature sensitivity of refractive index at $1550 \mathrm{~nm}$. Fig. 10(a) shows the sensitivity of the refractive index against the temperature change $(\mathrm{d} n / \mathrm{d} T)$ at the wavelength of $1550 \mathrm{~nm}$ at various values of $\mathrm{Al}$

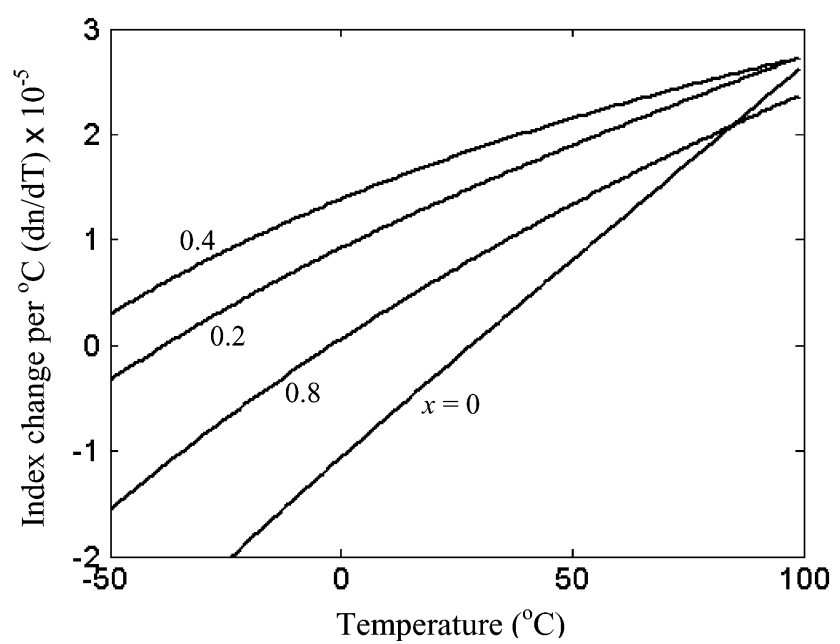

(a)

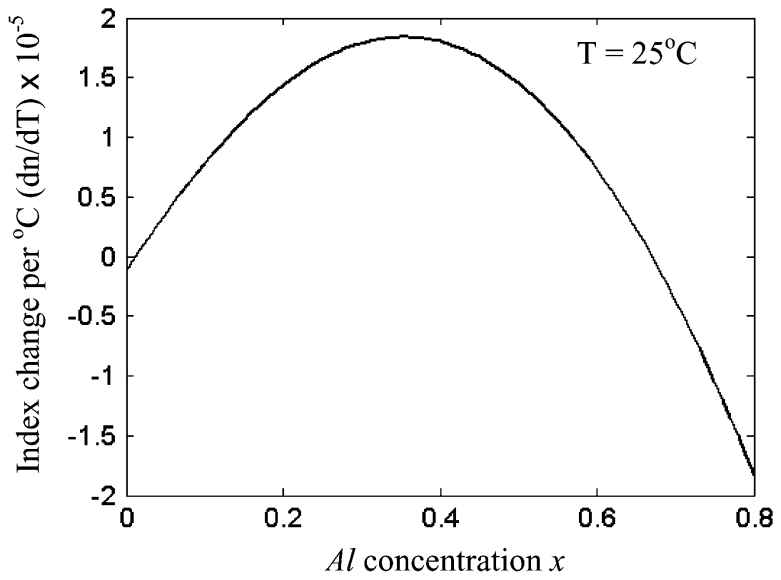

(b)

Fig. 10. Calculated temperature sensitivity of $\mathrm{AlGaN}$ refractive index (a) as a function of operation temperature for different $\mathrm{Al}$ concentration $x$ and (b) as a function of $\mathrm{Al}$ concentration with a fixed operating temperature at $25^{\circ} \mathrm{C}$.

concentration $x$. Within $0^{\circ}$ and $+50^{\circ} \mathrm{C}$, the temperature sensitivity is less than $2 \times 10^{-5}{ }^{\circ} \mathrm{C}$. This value is similar to that of silica [28] and approximately one order of magnitude lower than that of InP, which is about $3 \times 10^{-4}{ }^{\circ} \mathrm{C}$ [29], [30] at this wavelength. It is interesting to note that for $\mathrm{Al}_{x} \mathrm{Ga}_{1-x} \mathrm{~N}$ at room temperature $\left(25^{\circ} \mathrm{C}\right)$, the temperature sensitivity of refractive index is not monotonic versus $\mathrm{Al}$ concentration $x$, as shown in Fig. 10(b), it has a maximum around $x=0.4$.

\section{B. Efficiency of Carrier-Induced Index Change}

Carrier-induced refractive index change is the basic tuning mechanism for PHASAR based optical switches, which can be much faster than thermal tuning. So far, the efficiency of carrier-induced index change of $\mathrm{GaN}$ semiconductor in the infrared wavelength region, which is far away from the material bandgap, has not been fully investigated. To analyze this efficiency, we consider three major carrier effects, namely bandfilling, bandgap shrinkage, and free-carrier absorption.

Band filling is the decrease of optical absorption at energies slightly above the nominal bandgap due to impurity doping 
and free-carrier injection. When parabolic energy bands are assumed, for a direct-bandgap semiconductor, the optical absorption near the bandgap is given by the square-root law [31]

$$
\alpha_{0}(E)= \begin{cases}\frac{C}{E} \sqrt{E-E_{g}}, & E \geq E_{g} \\ 0, & E<E_{g}\end{cases}
$$

where $E=h \omega$ is the photon energy, $E_{g}$ is the bandgap energy, and $C$ is a constant.

Due to bandfilling, optical absorption is a function of the carrier concentration. We denote carrier-induced change in absorption coefficient as

$$
\Delta \alpha(N, P, E)=\alpha(N, P, E)-\alpha_{0}(E)
$$

where $\alpha(N, P, E)$ represents absorption coefficients in doped semiconductor and with carrier injection while $\alpha_{0}$ is the absorption of intrinsic materials in the absence of injection. $N$ and $P$ are the concentrations of free electrons and holes. Since the relationship between refractive index $n$ and the absorption coefficient $\alpha$ is

$$
n(E)=1+\frac{2 c \hbar}{e^{2}} \Re \int_{0}^{\infty} \frac{\alpha\left(E^{\prime}\right)}{E^{\prime 2}-E^{2}} d E^{\prime}
$$

where $c$ is the speed of light, $e$ is the electron charge, and $R$ indicates the principle value of the integral. The change of the refractive index caused by bandfilling can thus be expressed as

$$
\Delta n(N, P, E)=\frac{2 c \hbar}{e^{2}} \Re \int_{0}^{\infty} \frac{\Delta \alpha\left(N, P, E^{\prime}\right)}{E^{\prime 2}-E^{2}} d E^{\prime}
$$

Since in all cases bandfilling decreases the absorption coefficient at a fixed energy, the refractive index of the material also decreases due to bandfilling.

On the other hand, bandgap shrinkage is caused only by injected free carriers but not by doped carriers. The increase of free-carrier concentration due to current injection decreases the energy of the conduction band and increases the energy of the valence band. This is caused by the electron wave functions overlap, where a gas of interacting electrons with lower energy level is formed at the bottom of the conduction band while a gas of interacting holes with higher level is formed at the top of the valence band. This causes the shrinkage of the bandgap, which generates a red shift of the absorption curve. The following model can be adopted for this shrinkage

$$
\Delta E_{g}(x)= \begin{cases}\frac{\kappa}{\varepsilon_{s}}\left(1-\frac{x}{x_{c}}\right)^{1 / 3}, & x \geq x_{c} \\ 0, & x<x_{c}\end{cases}
$$

where $\kappa$ is a fitting parameter, $x_{c}$ is the critical concentration of the free carriers, and $\varepsilon_{s}$ is the relative static dielectric constant. Basically, the bandgap shrinkage happens when the injected free
TABLE I

VALUES OF SEMICONDUCTOR GaN PARAMETERS $(T=300 \mathrm{~K})$

\begin{tabular}{l|l}
\hline$E_{g}(\mathrm{eV})$ & 3.39 \\
\hline$C\left(\mathrm{~cm}^{-1} \cdot \mathrm{s}^{-1 / 2}\right)$ & $2.8 \times 10^{13}$ \\
\hline $\mathcal{E}_{s}$ & 8.9 \\
\hline$n$ & 2.335 \\
\hline$m_{e}\left(m_{0}\right)$ & 0.2 \\
\hline$m_{h h}\left(m_{0}\right)$ & 1.4 \\
\hline$m_{l h}\left(m_{0}\right)$ & 0.3 \\
\hline$m_{d h}\left(m_{0}\right)$ & 1.49 \\
\hline$N_{c}\left(\mathrm{~cm}^{-3}\right)$ & $2.2429 \times 10^{18}$ \\
\hline$N_{v}\left(\mathrm{~cm}^{-3}\right)$ & $4.5659 \times 10^{19}$ \\
\hline$x_{c}\left(\mathrm{~cm}^{-3}\right)$ & $6.6 \times 10^{18}$ \\
\hline$\kappa$ & 0.13 \\
\hline
\end{tabular}

carriers have such a large concentration that the correlation effects among the free carriers become significant. The change in the absorption due to band shrinkage is

$$
\Delta \alpha(\chi, E)=\frac{C}{E} \sqrt{E-E_{g}-\Delta E_{g}(\chi)}-\frac{C}{E} \sqrt{E-E_{g}} .
$$

Equation (18) predicts that $\Delta \alpha$ is always positive with the largest value near the bandgap because $\Delta E_{g}$ is negative according to (17). Based on the change of the absorption coefficient, the change in the refractive index caused by bandgap shrinkage can also be calculated by applying (16).

The third carrier effect in a semiconductor is free-carrier absorption or the plasma effect. A free carrier can absorb a photon and move to a higher energy level within the conduction band. The change in refractive index caused by this effect can be calculated with [31],

$$
\Delta n=\frac{-6.9 \times 10^{-22}}{n E^{2}}\left\{\frac{N}{m_{e}}+P\left(\frac{m_{\mathrm{hh}}^{1 / 2}+m_{\mathrm{lh}}^{1 / 2}}{m_{\mathrm{hh}}^{3 / 2}+m_{\mathrm{lh}}^{3 / 2}}\right)\right\}
$$

where $n$ is the refractive index of the material and $m_{e}, m_{\mathrm{hh}}$ and $m_{\mathrm{lh}}$ are the effective masses of electrons, heavy holes, and light holes, respectively.

The three carrier effects mentioned above were assumed to be mutually independent. A simple summation of these effects can be used to estimate the total change in the refractive index. The parameters used in our numerical calculation are listed in Table I, these parameters were extracted from [32] for $\mathrm{GaN}$ semiconductor.

Fig. 11 shows the index changes versus signal wavelength caused by bandfilling (a), band shrinkage, (b) and free-carrier absorption (c). The calculations were performed at three different carrier injection levels $N=7 \times 10^{18} \mathrm{~cm}^{-3}$ (solid line), $3 \times 10^{19} \mathrm{~cm}^{-3}$ (dashed line), and $6 \times 10^{19} \mathrm{~cm}^{-3}$ (dotted line). For band filling and bandgap shrinkage, carrier-induced index changes are largely anti-symmetric around the material bandgap, both values are reduced significantly with the increase of wavelength away from the bandgap. In the infrared wavelength region, free-carrier absorption is the dominant effect for carrier-induced refractive index change.

Fig. 12 shows the combined effect of carrier-induced index change versus signal wavelength and the index change at 


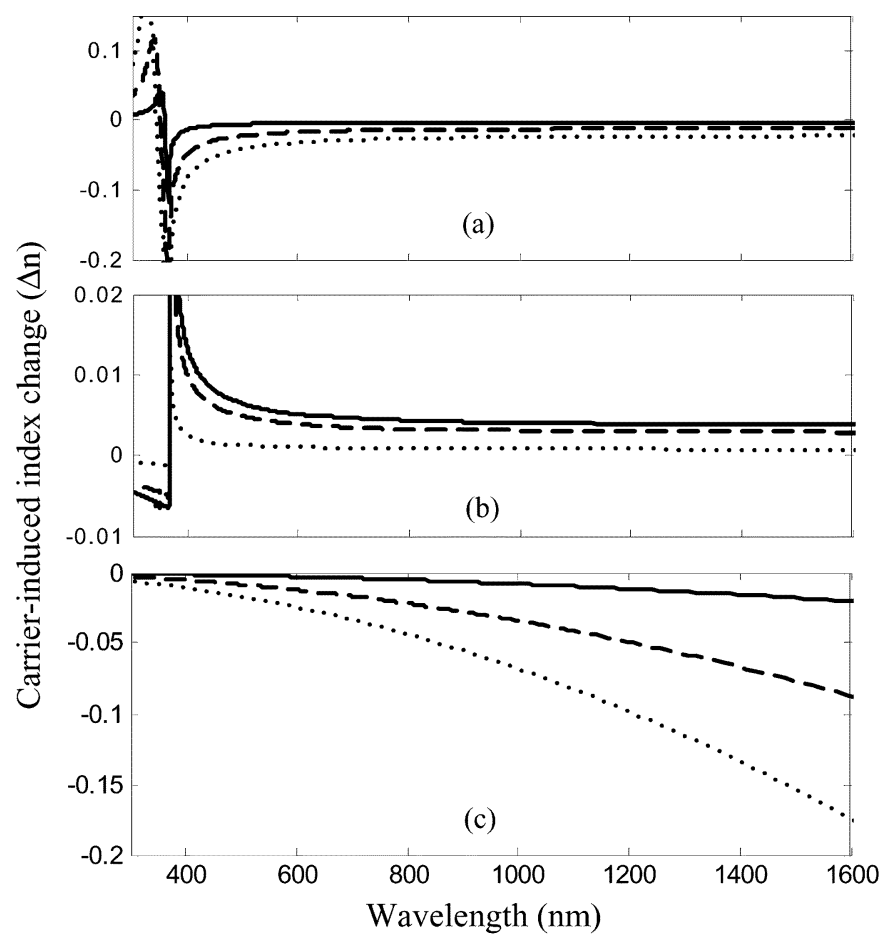

Fig. 11. (a) Index change due to bandfilling. (b) Bandgap shrinkage. (c) Free-carrier absorption. Solid line: $N=7 \times 10^{18} \mathrm{~cm}^{-3}$. Dashed line: $N=3 \times 10^{19} \mathrm{~cm}^{-3}$. Dotted line: $N=6 \times 10^{19} \mathrm{~cm}^{-3}$.
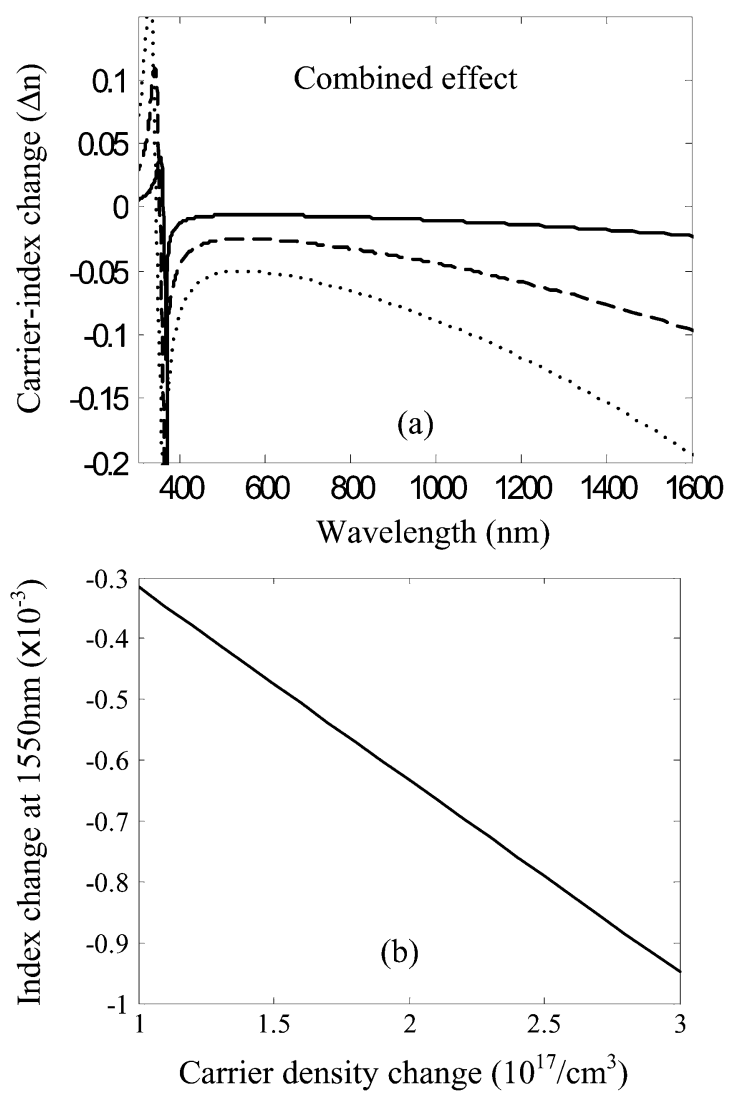

Fig. 12. (a) Overall index change versus wavelength calculated at three carrier density levels: $N=7 \times 10^{18} \mathrm{~cm}^{-3}$ (solid line), $3 \times 10^{19} \mathrm{~cm}^{-3}$ (dashed line), and $6 \times 10^{19}$, (dotted line). (b) Overall index change versus carrier density calculated at $1550-\mathrm{nm}$ wavelength. 1550-nm wavelength versus carrier concentration. In infrared wavelengths, far away from material bandgap, the refractive index change increases monotonically with the wavelength mainly due to free-carrier absorption.

For optical PHASAR applications, the index change of the waveguide should be large enough such that the optical length can be varied by up to a half wavelength of the optical signal [21]. Consider a $1 \mathrm{~mm}$ electrode on the waveguide and an optical signal at $1550-\mathrm{nm}$ wavelength. The minimum required index change should be approximately $0.0775 \%$. This can be achieved by a carrier density change of $2.5 \times 10^{17} \mathrm{~cm}^{-3}$, as indicated by Fig. 12(b), which is generally feasible. The numerical values obtained through this study provide us with important guideline for optical device design. The comparative study of different contributing effects is important for future device optimization for many potential applications.

\section{CONCLUSION}

In this paper, we have proposed the application of III-nitride semiconductor materials in infrared wavelength regions for fiber-optic communications. We have demonstrated the feasibility of making functional waveguide optical devices using $\mathrm{GaN}-\mathrm{AlGaN}$ heterostructures, such as straight waveguides, 3-dB optical waveguide couplers and AWG wavelength routers. The birefringence of wurtzite $\mathrm{GaN}$ grown on sapphire substrate has been observed. Refractive indexes were found to be different for signal optical field perpendicular or parallel to the crystal $c$ axis $\left(n_{\perp} \neq n_{/ /}\right)$. More importantly, we found an approximately $10 \%$ change in the index difference $\Delta n=n_{/ /}-n_{\perp}$ with varying the waveguide orientation within the $c$ plane, and a $60^{\circ}$ periodicity was clearly observed. This is attributed to the hexagonal structure of the nitride materials. The effect of birefringence needs to be overcome in practical integrated optical circuits applications, which may be accomplished by introducing built-in stresses in the crystal structure.

Thermal sensitivity of refractive index of $\mathrm{AlGaN}$ in the 1550-nm wavelength region has been investigated and has been found to be comparable to that of silica and approximately one order of magnitude lower than that of InP. The efficiency of carrier-induced refractive change in $\mathrm{GaN}$ has also been investigated considering bandfilling, bandgap shrinkage and free-carrier absorption. The results indicate that in infrared wavelengths, the dominant cause of carrier-induced refractive change is due to free-carrier absorption. The magnitude of carrier-induced refractive change is high enough for future applications as PHASAR devices for optical communications. It needs to be pointed out that although the application of III-nitrides for fiber-optic communications is promising, there is still much work to be done before it become practical, such as significant loss reduction through the improvement of material quality and the optimization of the photolithographic process as well as the reduction of the birefringence effect.

\section{REFERENCES}

[1] C. Dragone, "An $N \times N$ optical multiplexer using a planar arrangement of two star couplers," IEEE Photon. Technol. Lett., vol. 3, no. 9, pp. 812-815, Sep. 1991. 
[2] M. Okuno, K. Kato, Y. Ohmori, M. Kawachi, and T. Matsunaga, "Improved $8 \times 8$ integrated optical matrix switch using silica-based plannar lightwave circuits," J. Lightw. Technol., vol. 12, no. 9, pp. 1597-1605, Sep. 1994.

[3] N. Takato, T. Kominato, A. Sugita, K. Jingugi, H. Toba, and M. Kawachi, "Silica-based integrated optical Mach-Zehnder multi/demultiplexer family with channel spacing of 0.01-250 nm," IEEE Sel. Areas Commun., vol. 8, no. 6, pp. 1120-1127, Dec. 1990.

[4] C. R. Doerr, C. H. Joyner, L. W. Stulz, and R. Monnard, "Wavelengthdivision multiplexing cross connect in InP," IEEE Photon. Technol. Lett., vol. 10, no. 1, pp. 117-119, Jan. 1998.

[5] O. Wada and H. Hasegawa, Eds., InP-Based Materials and Devices, Physics and Technology. New York: Wiley, 1999. Sec. 2.4.

[6] S. Nakamura and G. Fasol, Eds., The Blue Laser Diodes. New York: Springer-Verlag, 1997.

[7] T. N. Oder, J. Y. Lin, and H. X. Jiang, "Propagation properties of light in AlGaN/GaN quantum-well waveguides," Appl. Phys. Lett., vol. 79, pp. 2511-2513, 2001

[8] R. Hui, S. Taherion, Y. Wan, J. Li, S. X. Jin, J. Y. Lin, and H. X. Jiang, "GaN-based waveguide devices for long wavelength optical communications,” Appl. Phys. Lett., vol. 82, no. 9, pp. 1326-1328, 2003.

[9] U. Tisch, B. Meyler, O. Katz, E. Finkman, and J. Salzman, "Dependence of the refractive index of $\mathrm{Al}_{x} \mathrm{Ga}_{1-x} \mathrm{~N}$ on temperature and composition at elevated temperatures," J. Appl. Phys., vol. 89, no. 5, pp. 2676-2685, 2001.

[10] K. B. Nam, J. Li, M. L. Nakarmi, J. Y. Lin, and H. X. Jiang, "Achieving highly conductive AlGaN alloys with high Al contents," Appl. Phys. Lett., vol. 81, pp. 1038-1040, 2002.

[11] D. Brunner, H. Angerer, E. Bustarret, F. Freudenberg, R. Höpler, R. Dimitrov, O. Ambacher, and M. Stutzmann, "Optical constants of epitaxial AlGaN films and their temperature dependence," J. Appl. Phys., vol. 82, pp. 5090-5096, 1997.

[12] V. Fiorentini, F. Bernardini, F. D. Sala, A. Di Carlo, and P. Lugli, "Effects of macroscopic polarization in III-V nitride multiple quantum wells," Phys. Rev. B, vol. 60, pp. 8849-88 582, 1999.

[13] S. Ghosh, P. Waltereit, O. Brandt, H. T. Grahn, and K. H. Ploog, "Polarization-dependent spectroscopic study of $M$ plane $\mathrm{GaN}$ on $-\mathrm{LiAlO}_{2}$," Appl. Phys. Lett., vol. 80, pp. 413-415, 2002.

[14] T. N. Oder, J. Y. Lin, and H. X. Jiang, "Fabrication and optical characterization of III-nitride sub-micron waveguides," Appl. Phys. Lett., vol. 79, pp. 12-14, 2001.

[15] M. Zirngibl, C. H. Joyner, and P. C. Chou, "Polarization compensated waveguide grating router on InP," Inst. Elect. Eng Electron. Lett., vol. 31, no. 19, pp. 1662-1664, 1995.

[16] H. Bissessur, P. Pagnod-Rossiaux, R. Mestric, and B. Martin, "Extremely small polarization independent phased-array demultiplexers on InP," IEEE Photon. Technol. Lett., vol. 8, no. 4, pp. 554-556, Mar. 1998.

[17] J. Sarathy, S. Chandrasekhar, A. D. Dentai, and C. R. Doerr, "Polarization insensitive waveguide grating routers in InP," IEEE Photon. Technol. Lett., vol. 8, no. 4, pp. 554-556, Mar. 1998.

[18] IEEE J. Quantum Electron., Special Issue on Strained-layer Optoelectronic Materials and Devices, vol. 30, no. 2, 1994.

[19] T. Kakitsuka, Y. Shibata, M. Itoh, Y. Kadota, Y. Tohmori, and Y. Yoshikuni, "Influence of buried structure on polarization sensitivity in strained bulk semiconductor optical amplifiers," IEEE J. Quantum Electron., vol. 38, no. 1, pp. 85-92, Jan. 2002.

[20] J. Li, K. B. Nam, M. L. Nakarmi, J. Li, J. Y. Lin, H. X. Jiang, P. Carrier, and S. H. Wei, "Band structure and fundamental optical transitions in Wurtzite AlN," Appl. Phys. Lett., vol. 83, pp. 5163-5165, 2003.

[21] M. K. Smit and C. van Dam, "PHASAR-based WDM-devices: Principles, design, and applications," IEEE J. Sel. Top. Quantum Electron., vol. 2, no. 2, pp. 236-250, Jan. 1996.

[22] R-Soft BPM Simulation Manual, R-Soft, Inc., Ossining, NY.

[23] Y. Chen, R. Schnrider, S. Y. Wang, R. S. Kern, C. H. Chen, and C. P. Kuo, "Dislocation reduction in GaN thin films via lateral overgrowth from trenches," Appl. Phys. Lett., vol. 75, pp. 2062-2064, 1999.

[24] C. H. Ashby, C. C. Mitchell, J. Han, N. A. Missert, P. P. Provencio, D. M. Follstaedt, G. M. Peake, and L. Griego, "Low-dislocation-density GaN from a single growth on a textured substrate," Appl. Phys. Lett., vol. 77, pp. 3233-3235, 2000

[25] H. Amano, M. Iwaya, S. Nitta, S. Terao, R. Nakamura, T. Ukai, S. Saitoh, S. Kamiyama, C. Wetzel, and I. Akasaki, "Defect and stress control of AlGaN and fabrication of high-effiiency UV-LED," in Proc. Mat. Res. Soc. Symp. , vol. 639, 2000, Paper No. G12.71.
[26] J. Li, K. B. Nam, M. L. Nakarmi, J. Y. Lin, and H. X. Jiang, "Bandedge photoluminescence of AlN epilayers," Appl. Phys. Lett., vol. 81, pp. 3365-3367, 2002

[27] K. B. Nam, J. Li, M. L. Nakarmi, J. Y. Lin, and H. X. Jiang, "Deep ultraviolet picosecond time-resolved photoluminescence studies of AlN epilayers," Appl. Phys. Lett., vol. 82, pp. 1694-1696, 2003.

[28] I. H. Malitson, "Interspecimen comparison of the refractive index of fused silica," J. Opt. Soc. Amer, vol. 55, pp. 1205-1209, 1965.

[29] M. Bertolotti, V. Bodanov, A. Ferrari, A. Jascow, N. Nazorova, A. Pikhtin, and L. Schirone, "Temperature dependence of the refractive index in semiconductors," J. Opt. Soc. Amer. B., vol. 7, pp. 918-922, 1990.

[30] P. Martin, E. Mostafa, L. Chusseau, and C. Alibert, "Accurate refractive index measurements of doped and undoped InP by a grating coupling technique," Appl. Phys. Lett., vol. 67, no. 7, pp. 881-883, 1995.

[31] B. R. Bennett, R. A. Soref, and J. A. Edl Alamo, "Carrier-induced change in refractive index of InP, GaAs, and InGaAsP," IEEE $J$. Quantum Electron., vol. 26, no. 1, pp. 113-122, Jan. 1990.

[32] V. Bougrov, M. Levinshtein, S. Rumyantsev, and A. Zubrilov, "Gallium Nitride (GaN)," in Properties of Advanced Semiconductor Materials, GaN, AlN, InN, BN, SiC, SiGe, M. E. Levinshtein, S. L. Rumyantsev, and M. S. Shur, Eds. New York: Wiley, 2001.

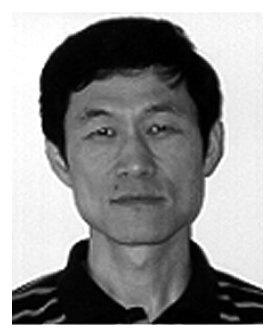

Rongqing Hui (M'97-SM'97) received the B.S. degree in microwave communications in 1981 and the M.S. degree in lightwave technology in 1988, both from Beijing University of Posts and Telecommunications, Beijing, China. He received the Ph.D. degree in electrical engineering from Politecnico di Torino, Torino, Italy, in 1993

From 1982 to 1985 , he taught at the Physics Department of Anhui University, Hefei, China, where he also conducted research on optical fibers and fiber sensors. From 1985 to 1989, he was with the Optical Communication Laboratory of Beijing University of Posts andTelecommunications, where he worked in coherent optical fiber communication systems and components. From 1989 to 1990, he held a research fellowship from the Fundazione Ugo Bordoni, Rome, Italy, working on nonlinear effects and optical injection locking of semiconductor laser devices. From 1990 to 1993, he was with the Department of Electronics, Politecnico di Torino, where he worked on optical communications and single frequency semiconductor laser devices. During this period, he also worked as a Research Engineer at the Telecommunication Research Center (CSELT), Torino, Italy. He spent one year, from 1993 to 1994, as a Postdoctoral Research Fellow working on optical systems \& networks architecture at the University of Ottawa, ON, Canada. He joined Bell-Northern Research (now part of Nortelnetworks), Ottawa, Canada, in 1994 as a Member of Scientific Staff, where he has worked in the research and development of high-speed optical transport networks. Since September 1997, he has been with the department of Electrical Engineering and Computer Science, University of Kansas, Lawrence, where he is a tenured Associate Professor. As an author or co author, he has published more than 70 refereed technical papers in the area of fiber-optic communications and holds 11 patents.

Dr. Hui currently serves as an Associate Editor of IEEE TRANSACTIONS ON COMMUNICATIONS.

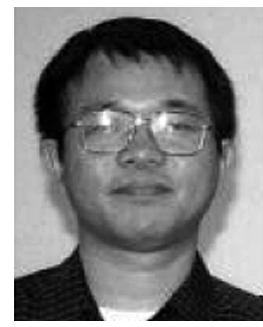

Yueting Wan received the B.S. degree in physics from the University of Science and Technology of China, Anhui, China, in 1987, the M.Eng. degree in telecommunications from the Asian Institute of Technology, Bangkok, Thailand, in 1997, M.S. in physics from University of Memphis, Memphis, TN, in 1999 . He is currently working towards the Ph.D. degree in electrical engineering at the University of Kansas, Lawrence.

He had been teaching college physics at Jiangsu University of Science and Technology, Jiangsu, China, from 1987 to 1996. His research interests include all-optical switch design and testing and the research of novel semiconductor material properties in the applications of fiber-optic telecommunications. 


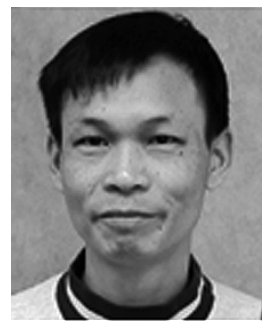

Jing $\mathbf{L i}$ received the B.S. degree in applied physics from Wuhan University, China, in 1984, the M.S. degree from the Ministry of Electronic Industries Hebei Semiconductor Research Institute, Hebei, China, in 1987, and the Ph.D. degree in physics in 2003 from Kansas State University.

$\mathrm{He}$ is a Senior Scientist at III-N Technology Incorporated, Manhattan, KS, and is currently a Visiting Senior Scientist at Kansas State University, Lawrence. He has published over 50 journal papers and holds three patents on nitride technology.

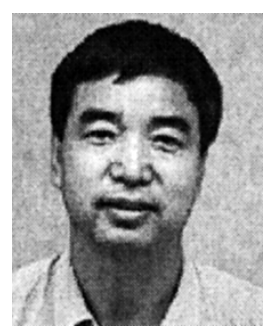

Sixuan Jin is currently a Member of Research Staff, Kansas State University (KSU), Manhattan. He was a Senior Research Engineer of Peking University, Peking, Ching, prior to joining KSU.

He has published 45 journal papers and holds three patents on nitride technology.

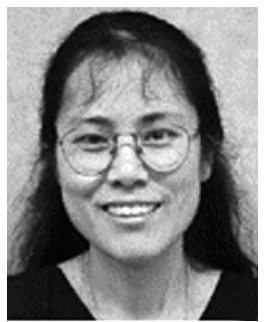

Jingyu Lin received the B.S. degree in physics from the State University of New York College at Oneonta in 1983 and the Ph.D. degree in physics from Syracuse University, Syracuse, NY, in 1989.

She is currently is a Full Professor of Physics at Kansas State University, Manhattan. Her current research dedicates to the advancements of III-nitride wide bandgap semiconductor physics and technologies. She has organized national and international workshops and symposia, delivered numerous invited presentations in national and international conferences, and served as a review panelist for many national interdisciplinary research programs. She has published over 200 refereed journal papers, written several book chapters, and holds six patents on III-nitride technology.

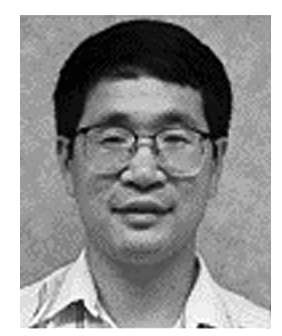

Hongxing Jiang received the B.S. degree in physics from Fudan University, Shanghai, China, in 1981 and the Ph.D. degree in physics from Syracuse University, Syracuse, NY.

$\mathrm{He}$ is currently a University Distinguished Professor of Physics at Kansas State University, Mnahattan. His research is dedicated to the experimental investigations in material growth, device fabrication, and optical and electrical properties of III-nitride wide bandgap semiconductors. He has published 220 refereed journal papers in the area of compound semiconductors, holds 7 patents, edited 6 books, written 15 book chapters and review articles, and delivered over 60 invited talks in professional conferences.

Dr. Jiang has served as Conference Chair or Co-chair for many international conferences and workshops. 